\title{
Streptococcus pneumoniae - an uncommon cause of neonatal sepsis: two case reports
}

\author{
GCS Gunasekera $^{1}$, GPS Gunaratna ${ }^{2}$, R Sathanantharajah ${ }^{3}$, NS Chandrasiri ${ }^{3}$ \\ Sri Lankan Journal of Infectious Diseases 2022 Vol.12(1):E5 1-6 \\ DOI: http://dx.doi.org/10.4038/sljid.v12i1.8420
}

\begin{abstract}
Streptococcus pneumoniae is a rare but recognized aetiological agent for neonatal sepsis. In the context of scarce local data, we report two cases of early-onset neonatal sepsis (EONS) due to pneumococci that probably occurred following ascending transmission during the perinatal period.
\end{abstract}

Keywords: Neonatal sepsis, early-onset neonatal sepsis (EONS), Streptococcus pneumoniae, pneumococci, colonization

\section{Introduction}

Streptococcus pneumoniae is a well-recognized but uncommon cause of EONS with high morbidity/mortality. The estimated incidence of pneumococcal neonatal sepsis is $1 \%-10 \%$ of all neonatal sepsis. ${ }^{1}$ Sri Lankan data on pneumococcal neonatal sepsis is scarce. The newborn acquires the infection as an ascending infection from the colonized maternal vagina or haematogenously via the placenta. Prolonged rupture of membranes, maternal genital tract colonization, prematurity and low birth weight are associated factors. ${ }^{2,3 .}$

We report two cases of pneumococcal EONS that probably occurred following ascending transmission during the perinatal period. We would like to note that blood cultures and identifications of microorganisms were done using manual methods.

\footnotetext{
${ }^{1}$ Department of Microbiology \& Immunology, Faculty of Medicine, University of Colombo, Sri Lanka

${ }^{2}$ Department of Parasitology, Faculty of Medicine, University of Kelaniya, Sri Lanka

${ }^{3}$ Colombo South Teaching Hospital, Kalubowila, Sri Lanka

Address for correspondence: Dr GCS Gunasekera, Department of Microbiology \& Immunology, Faculty of Medicine, University of Colombo, Sri Lanka Telephone: +0094714934525 E-mail: chathurigcs@yahoo.com

(iD https://orcid.org/0000-0002-5141-6319

Received 12 October 2021 and revised version accepted 22 January 2022. Published 25.2.22

(c) (1) use, distribution, and reproduction in any medium, provided the original author and source are credited.
} 


\section{Case 1}

A 19-year-old primigravida was admitted at 35 weeks and 6 days of gestation, with dribbling for one hour. Clinical examination showed no evidence of chorioamnionitis. She was started on oral erythromycin and intramuscular dexamethasone. After 24 hours of dribbling, she developed fever of $100{ }^{\circ} \mathrm{F}$ and lower abdominal pain. Her white cell count went up to $34000 / \mathrm{mm}^{3}(86 \%$ neutrophils) from $11600 / \mathrm{mm}^{3}$ but her C-reactive protein and urine tests were normal. The antibiotic was changed to intravenous cefuroxime and metronidazole. A high vaginal swab (HVS) had a heavy growth of $\beta$-haemolytic Streptococcus spp. sensitive to penicillin and erythromycin but resistant to clindamycin which was reported as probable Group B streptococci (GBS). This was based on the local laboratory policy, in which all bile aesculin negative streptococci which grew on MacConkey agar were reported as probable Group B Streptococci.

On day four of admission, the mother delivered a baby girl with a birth weight of $2.4 \mathrm{~kg}$ by normal vaginal delivery (NVD). Cyanosed at birth with Apgar score of $1^{0} 5^{3} 10^{3}$, the baby was resuscitated and intubated and transferred to the special care baby unit (SCBU). Benzyl penicillin and gentamicin were started intravenously after taking blood cultures. The baby went into severe respiratory distress and was ventilated. Intercostal tubes were inserted due to bilateral pneumothorax. Appropriate supportive care was given; inotropes were started. However, the baby succumbed to her illness at 7 hours of age, accounting for an early neonatal death. A pathological post-mortem revealed intracerebral (sub-arachnoid and intraventricular) haemorrhages due to severe birth asphyxia. A retrospective inquiry into the records of the HVS culture showed evidence of a mixed growth of streptococci, though it had been reported as probable Group B streptococci. However, it was not possible to obtain a fresh HVS sample for retesting.

The blood culture was positive for $S$. pneumoniae after 24 hours of incubation. The isolate was identified by biochemical methods (alpha haemolysis, susceptibility to optochin and bile solubility). It is worth noting that bile solubility was checked in all optochin susceptible streptococcal isolates, taking into consideration that optochin-resistant Streptococcus pneumoniae has been reported in Sri Lanka. ${ }^{4}$ The isolate was sensitive to penicillin with a minimum inhibitory concentration (MIC) of $<0.06 \mu \mathrm{g} / \mathrm{ml}$, cefotaxime, vancomycin and chloramphenicol. Antibiotic susceptibilities were done by the disc diffusion method and e-strip MIC for penicillin. The mother was discharged 2 days post-partum, following counselling and suppression of lactation.

The timeline of this patient's clinical course is given in Figure 1 


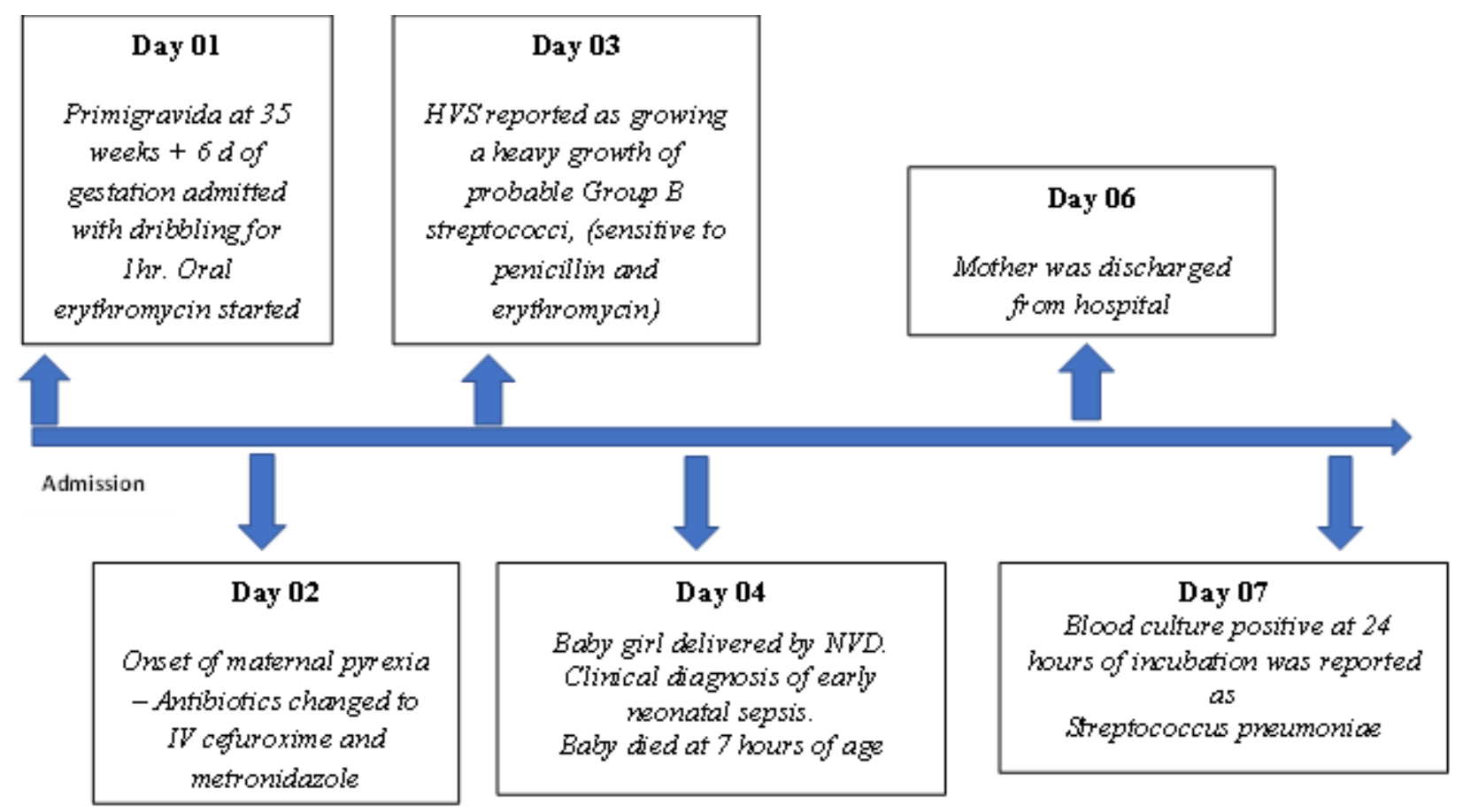

Figure 1: Timeline of clinical course of Case 1

Case 2

A 35-year-old mother $\left(\mathrm{P}_{2} \mathrm{C}_{1}\right)$ at 39 weeks and 5 days of gestation was admitted with abdominal pain and dribbling for four hours without fever. Her antenatal period was uneventful. A live nonasphyxiated baby girl with a birth weight of $3.1 \mathrm{~kg}$ was delivered by NVD. Ten hours later, the baby became tachypnoeic with low oxygen saturation. The baby was transferred to the SCBU. Intravenous penicillin and cefotaxime were started after obtaining blood for culture and other investigations. However, the baby deteriorated rapidly over the next few hours. Her white cell count was raised and chest X-ray showed extensive bilateral inflammatory shadows.. Despite appropriate care and ventilation, the baby died at 36 hours of birth.

The blood culture grew S. pneumoniae after 24 hours of incubation which was sensitive to penicillin (MIC was $<0.06 \mu \mathrm{g} / \mathrm{ml}$ - sensitive according to both meningitis and non-meningitis breakpoints), erythromycin, clindamycin, cefotaxime, vancomycin and chloramphenicol. Antibiotic susceptibilities were done by the disc diffusion method and e-strip MIC for penicillin. The isolate was identified by biochemical methods (alpha haemolysis, susceptibility to optochin and bile solubility). The latter was done despite optochin susceptibility as optochin-resistant Streptococcus pneumoniae has been reported in Sri Lanka. ${ }^{4}$ Retrospective review of the worksheet of the mother's HVS culture showed that alpha-haemolytic streptococci were isolated. However, further identification had not been carried out.

The timeline of this patient's clinical course is given in Figure 2 


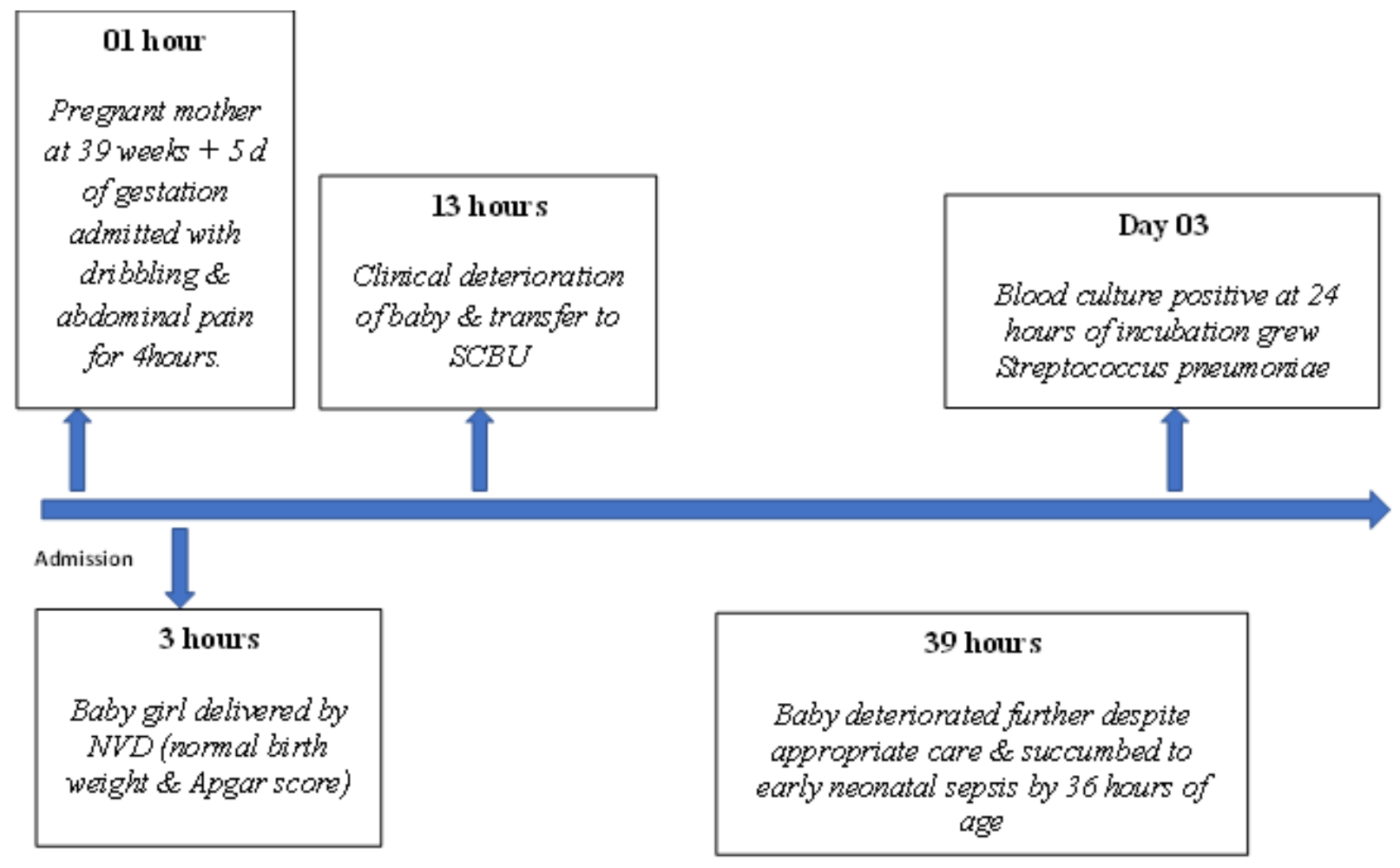

Figure 2: Timeline of clinical course of Case 2

\section{Discussion}

Streptococcus pneumoniae is a rare cause of severe invasive neonatal sepsis. It accounts for $1 \%$ to $5.5 \%$ of neonatal sepsis cases in the United States and Europe and for $11.5 \%$ in developing countries. $^{2}$

The usual mode for a newborn to acquire this infection is through ascending infection from the colonized maternal genital tract. The normal vaginal microbiome does not carry $S$. pneumoniae as it is unable to survive in the acidic environment of the vagina. However, during pregnancy, the vagina becomes less acidic, thus providing a better environment for colonization by pneumococci. ${ }^{3}$ The incidence of vaginal colonization in pregnant women is low $(0.03 \%-0.75 \%$ of cases). ${ }^{3,5}$ Possible ways of colonization by pneumococci are through oro-genital sexual practices, use of contaminated instruments such as specula and the use of contaminated gloves. ${ }^{3}$ Pneumococcal neonatal sepsis secondary to maternal endometritis has also been described. ${ }^{5}$ Haematogenous or transplacental transmission of pneumococci is a recognized mode of acquiring neonatal sepsis. ${ }^{3}$

The above described two cases are infections probably acquired through the maternal genital tract and demonstrate the importance of identifying alpha-haemolytic streptococci isolated from vaginal swabs sent for Group B streptococcal screening. Identification of alpha-haemolytic streptococci isolated from the HVS samples of both mothers had been missed on both occasions. 
The lack of facilities required for routine identification of streptococci (streptococcal grouping kits, reliable biochemical methods for identification of alpha-haemolytic streptococci) contributed to the non-identification in both instances. These two case reports highlight the importance of providing such facilities to clinical microbiology laboratories in Sri Lanka.

Infected babies may present with poor feeding, excessive crying, less activity or evidence of systemic involvement such as respiratory distress or convulsions. ${ }^{6}$ Early and prompt diagnosis and optimum antibiotic management will reduce morbidity/mortality. Treatment of pneumococcal neonatal sepsis should be based on the antibiotic susceptibility. Pneumococci are generally sensitive to penicillin and cefotaxime ${ }^{7}$, which are the usual empirical choices in managing neonatal sepsis. According to the available local data on antibiotic susceptibility of pneumococcal isolates from patients of all ages, $91.3 \%$ of penicillin resistance is reported. Resistance to the third generation cephalosporins is also high $(47.83 \%){ }^{8}$ Though these publications were before the separation of meningeal/non-meningeal cut-offs by the Clinical Laboratory Standards Institute in 2008, meningeal MIC cut-offs are still valid. According to the standard antibiotic guidelines, the MIC expected for central nervous system (CNS) isolates are much lower than the MIC for blood culture isolates. In neonatal sepsis, CNS involvement is frequent. Therefore, knowing the MICs of the regional/local pneumococcal isolates is important in determining the empirical treatment in suspected cases of pneumococcal neonatal sepsis.

Prevention of neonatal pneumococcal infections is important in reducing its high morbidity/mortality. Pneumococcal neonatal sepsis could be due to vaccine serogroups or nonvaccine serogroups. Maternal vaccination, via passive immunization, will protect the neonate against the vaccine serogroups of pneumococci. ${ }^{9}$ However, the new Australian National Immunization Schedule which provides a 23-valent pneumococcal polysaccharide vaccine dose in late adulthood did not seem to protect females during their pregnancy. ${ }^{9}$ Vaccination during the third trimester of gestation could be a measure to follow in the future, although there are no conclusive studies that currently confirm this.

\section{Conclusion}

We describe two fatal EONS cases due to $S$. pneumoniae which is a rare cause of EONS. Better awareness about this pathogen is needed to detect it from maternal HVS. As Sri Lanka is not providing routine immunization against pneumococci, mothers may not have antibodies against most serotypes. There are only a few Sri Lankan studies done on pneumococcal serotypes which have been done on invasive isolates and respiratory colonization. ${ }^{8,10,11}$ Scarcity of data on serotypes, especially on colonizing flora is a gap in local knowledge.

\section{Declarations}

Acknowledgement: None

Funding source: No funding and other support obtained from any source

Conflict of Interest: There were no conflicts of interest in this study

Ethics statement: Ethical approval was not required for this manuscript.

Author contributions: All the authors contributed in-patient management and preparing manuscript. 


\section{References}

1. McDonald LC, Bryant K, Snyder J. Peripartum transmission of penicillin-resistant Streptococcus pneumoniae. J. Clin Microbiol. 2003; 41(5):2258-2260. doi: https://doi.org/10.1128/JCM.41.5.2258-2260.2003

2. Hoffman JA, Mason EO, Schutze GE et al. Streptococcus pneumoniae infections in the neonate. Pediatrics. 2003; 112(5):1095-1102. doi: https://doi.org/10.1542/peds.112.5.1095

3. Fernandez B, Luis R, Mascaraque LR et al. Streptococcus Pneumoniae: The forgotten microorganism in neonatal sepsis. Fetal and Pediatric Pathology. 2014; 34(3):202-205. Available at: https://streptococcuspathogen.blogspot.com/2015/05/streptococcus-pneumoniaeforgotten.html

4. Karunanayake L, Tennakoon C. Optochin-resistant Streptococcus pneumoniae. Ceylon Medical Journal. 2011; 56(2):84. doi: https://doi.org/10.4038/cmj.v56i2.3123

5. Dias MFA, Silva NR, Garces F et al. Neonatal serotype 8 pneumococcal invasive disease; A happy end in a potentially fatal disease. BAOJ Pediat. 2016; 2(3):2-016. No doi Available at: https://www.researchgate.net/publication/310607943

6. Hughes BR, Mercer Jl, Gosbel LB. Neonatal pneumococcal sepsis in association with fatal maternal pneumococcal sepsis. Aust. NZJ Obstet Gynaecol. 2001; 41(4):457-8. doi: https://doi.org/10.1111/j.1479-828x.2001.tb01331.x

7. Khalifa K, Agarwal M. Neonatal Streptococcus pneumoniae sepsis: rare but fatal. The Internet Journal of Pediatrics and Neonatology. 2004; 5(2):202-205. Available at: http://ispub.com/IJPN/5/2/6002

8. Batuwanthudawe R, Karunarathne K, Dassanayake M et al. Surveillance of invasive pneumococcal disease in Colombo, Sri Lanka. Clinical Infectious Diseases 2009; 48:136-40. doi: https://doi.org/10.1086/596492

9. Chaithongwongwatthana S, Yamasmit W, Limpongsanurak S. Pneumococcal vaccination during pregnancy for preventing infant infection. Cochrane Database Syst Rev. 2015;1: CD004903. doi: https://doi.org/10.1002/14651858.CD004903.pub4

10. Vidanapathirana G, Angulmaduwa S, Munasinghe, T et al. Pneumococcal colonization among healthy and hospitalized vaccine-naive Sri Lankan children. Vaccine. 2020; 38(46):7308-7315. doi: https://doi.org/10.1016/j.vaccine.2020.09.040

11. Watanabe H, Batuwanthudawe R, Thevanesam V et al. Possible prevalence and transmission of acute respiratory tract infections caused by Streptococcus pneumoniae and Haemophilus influenzae among the internally displaced persons in tsunami disaster evacuation camps of Sri Lanka. Internal Medicine, 2007; 46(17):1395-1402.

doi: https://doi.org/10.2169/internalmedicine.46.0149 